\title{
USO DE GEOTECNOLOGIAS PARA GESTÃO DE RECURSOS NATURAIS: caso do macrozoneamento ecológico econômico do Maranhão
}

\section{USE OF GEOTECHNOLOGIES FOR NATURAL RESOURCES MANAGEMENT: case of the economic ecological macrozonamento of the Maranhão}

\begin{abstract}
Érima Jôyssielly Mendonça Castro ${ }^{1}$, Adriano Oliveira Macedo ${ }^{2}$, Allyson Luis Ramos da Silva ${ }^{3}$, Thiago Frazão Talhari ${ }^{4}$, Denilson da Silva Bezerra ${ }^{5}$, Fabrício Silva Brito ${ }^{6}$.
\end{abstract}

RESUMO: A disponibilidade de recursos naturais limita o crescimento populacional e seu desenvolvimento. O uso e manejo inadequado provenientes das ações antrópicas refletem negativamente na disponibilidade desses recursos e na qualidade ambiental. Através da gestão ambiental é possível administrar a sua utilização de maneira sustentável, favorecendo tanto o crescimento econômico quanto a conservação dos recursos naturais. $\mathrm{O}$ uso de geotecnologias torna a gestão ambiental mais eficaz e eficiente, subsidiando-a com bases de dados espaciais que permitem avaliar o potencial ambiental e identificar possíveis conflitos no aproveitamento desse potencial quanto à utilização dos recursos naturais. Nos últimos anos a utilização do sensoriamento remoto e técnicas de geoprocessamento têm sido efetivas, principalmente no monitoramento ambiental do Brasil, possibilitando diagnósticos de áreas de difícil acesso por meio de transportes e de acordo com a resolução temporal dos satélites pode-se realizar um monitoramento satisfatório destas áreas mais restritas. O presente artigo objetiva propor uma gestão dos recursos naturais do Maranhão através do uso de dados espaciais. Buscando-se identificar e analisar ações de gestão ambiental aplicadas ao território maranhense. Para o estudo, utilizaram-se dados referentes a geotecnologias com o intuito de reconhecer as áreas prioritárias para conservação e a disposição hidrográfica. Foram feitas análises a partir da construção de mapas resultantes do Macrozoneamento Econômico Ecológico do Maranhão (macroZEE-MA). Obtiveram-se como resultados dados vetores e imagens que apontam que é possível realizar uma gestão ambiental capaz

\footnotetext{
${ }^{1}$ Graduanda do $7^{\circ}$ período de Engenharia Ambiental da Universidade Ceuma. E-mail: erima.castro@hotmail.com

${ }^{2}$ Graduando do $7^{\circ}$ período de Engenharia Ambiental da Universidade Ceuma. E-mail: adriano matt@hotmail.com

${ }^{3}$ Graduando do $7^{0}$ período de Engenharia Ambiental da Universidade Ceuma. E-mail: allyson.80@hotmail.com

4 Graduando do $7^{\circ}$ período de Engenharia Ambiental da Universidade Ceuma.E-mail: thiago_talhari@hotmail.com

5 Professor Doutor do Mestrado em Meio Ambiente e do curso de Engenharia Ambiental da Universidade Ceuma.

E-mail:denilson_ca@yahoo.com.br

6 Coordenador do Laboratório de Geotecnologias e Professor Doutor do curso de Engenharia Ambiental da Universidade Ceuma.

E-mail: fabricio.brito@,ceuma.br
} 
de assegurar sustentavelmente o maior aproveitamento do potencial ambiental nas suas possíveis destinações de uso junto à qualidade do meio.

PALAVRAS CHAVE: Geotecnologias; Macrozoneamento; Recursos Naturais.

ABSTRACT: The availability of natural resources limits population growth and development. The use and inadequate management of anthropogenic actions negatively reflect the availability of these resources and the environmental quality. Through environmental management it is possible to manage its use in a sustainable way, favoring both economic growth and conservation of natural resources. The use of geotechnologies makes environmental management more effective and efficient, subsidizing them with spatial data bases that allow to assess the potential environmental and identify possible conflicts in the exploitation of this potential with regard to the use of natural resources. In recent years the use of remote sensing and geoprocessing techniques have been effective mainly in environmental monitoring of Brazil, enabling diagnosis of areas of difficult access by means of transport, and in accordance with the temporal resolution of the satellites you can achieve a satisfactory monitoring of these areas more restricted. This article aims to propose a management of natural resources of Maranhão through the use of spatial data. Seeking to identify and analyze actions of environmental management applied to the territory of Maranhão. For the study, we used data referring to geotechnologies aimed to recognize the priority areas for conservation and the watershed provision. It was obtained as a result vector data and images that indicate that it is possible to carry out an environmental management capable of ensuring the most sustainably exploiting the environmental potential in their possible destinations for use beside the quality of the environment.

KEYWORDS: Geotechnology; Macro-Zoning; Natural Resources. 


\section{INTRODUÇÃO}

Segundo Malthus 1960, o crescimento das populações é limitado pelos recursos alimentares disponíveis e o aumento desses recursos não consegue acompanhar o crescimento populacional. Em seu ensaio sobre a população, destacava o crescimento populacional em progressão geométrica frente ao aumento dos recursos somente em progressão aritmética. Diante disso, a gestão não só dos recursos alimentares, mas dos recursos naturais em geral, se faz necessária para o desenvolvimento sustentável e repressão do esgotamento de recursos naturais:

"Pode-se seguramente declarar [...] que a população, quando não controlada, dobra a
cada 25 anos, ou aumenta numa razão geométrica. [...] Mas o alimento, para suportar o
aumento de um número tão grande, de nenhum modo será obtido com a mesma facilidade.
[...] Pode-se com justeza declarar [...] que, considerando-se na média o presente estado da
terra, os meios de subsistência, sob as condições mais favoráveis da indústria humana,
possivelmente não poderiam crescer mais rapidamente do que numa média aritmética." (MALTHUS, 1960)

A gestão dos elementos naturais em geral se faz necessária e indispensável para o desenvolvimento sustentável e repressão do esgotamento de recursos naturais. Infelizmente, os problemas causados pela ausência dessa gestão são encontrados comumente nos mais diversos cenários, em diferentes regiões e ecossistemas, afetando direta ou indiretamente a qualidade de vida do homem e dos demais seres vivos (FUKS, 1994).

A ação antrópica predatória, sucedida pela ausência da gestão e planejamento ambiental é uma das causas diretas do esgotamento e/ou perda da qualidade dos recursos, visto que o planejamento ambiental constitui um dos instrumentos fundamentais para a proteção dos mesmos e para prover orientação e subsídio à sua gestão, visando o gerenciamento dos recursos hídricos, terrestres e atmosféricos a partir de políticas públicas e ambientais, garantindo sustentabilidade ao desenvolvimento (LEMOS E SILVA, 2011).

Sem gestão e planejamento, o uso irracional e exploração descontrolada dos bens naturais tende a crescer, provocando impactos ambientais (CARDOSO E SABÓIA, 2013). O uso e ocupação de terras também é um dos principais problemas socioambientais, principalmente quando se trata de áreas de preservação permanente, por exemplo, pois tem como consequência dessa ação do homem limitar a quantidade de recursos naturais da área delimitada. A demanda aumenta proporcionalmente à expansão da população, resultando na degradação da qualidade das 
águas superficiais (NASCIMENTO et al., 2005). Outro fator que contribui para a exaustão dos recursos naturais é o desmatamento, diminui a biomassa e, consequentemente, reduz a capacidade de absorção de água pelo solo. Sem a cobertura vegetal o solo torna-se mais vulnerável à erosão e, exposto à luz solar, há um aumento no albedo da superfície, oxidando a matéria orgânica, causando a morte dos microrganismos pelas altas temperaturas, diminuindo a fertilidade do solo e levando à perda do horizonte superficial (SILVA et al., 2009). Os projetos de assentamentos de reforma agrária também se tornam uma ameaça. Isto porque, ao se consolidar um projeto de assentamento, os trabalhadores encontram diversas dificuldades e estas vão além das condições naturais desfavoráveis, como a falta de financiamento para projetos de recuperação, falta de acompanhamento técnico, burocracia para recebimento de crédito rural, serviços de saúde e educação precários, inexistência das vias de acesso, dentre outros (MEDEIROS, 1999). Isto força os agricultores a intensificar o uso das terras agrícolas e, como muitos dos assentados desconhecem técnicas conservacionistas de uso e manejo do solo, o utilizam de forma predatória (CAPONI E SANTOS, 2012).

Grande extensão geográfica, riqueza biológica e mineral e a diversidade de ecossistemas tornam o sensoriamento remoto uma ferramenta indispensável para estudo, permitindo o mapeamento e rápido monitoramento com baixo custo. Embora a legislação ambiental brasileira seja considerada bastante ampla, alguns fatores têm contribuído para torná-la pouco ágil (FILHO et al., 2006).

Técnicas de geoprocessamento e sensoriamento remoto se tornam cada vez mais essenciais para reconhecimento e monitoramento de áreas sob diversidade natural associados ao desenvolvimento e gestão da mesma, dispondo-a para uso adequado e sustentável. Um aspecto relevante é que o uso dessa opção tecnológica adquire maior importância à medida que o problema a ser analisado apresenta-se em grandes dimensões, complexidade e com custos para operacionalização em campo bastante elevados (BOLFE et al., 2004).

Na Amazônia, por exemplo, Imagens do satélite Landsat têm sido a principal fonte de dados para monitorar o avanço do desmatamento da região, por meio do Programa de Monitoramento do Desmatamento na Amazônia Legal (Prodes). Imagens do sensor MODIS estão sendo utilizadas pelo Inpe em um novo sistema de detecção de desmatamento em tempo real desde 2004 (FILHO et al., 2006). Segundo Bolfe et. al 2004, as imagens do satélite Landsat 5-TM são muito usadas para fazer estudos sobre a cobertura vegetal, pois possui características espectrais particulares nas faixas do vermelho e infravermelho próximo.

Elaboração de mapas de usos da terra com base nas imagens do satélite IKONOS II, 
auxiliam na delimitação de maneira automática das áreas de preservação permanente e identificar a ocorrência de conflito de uso (NASCIMENTO et al., 2005). Com uso de imagens de radar é possível realizar estudos florestais sob diferentes cenários. A elaboração de cartas sistemáticas, materiais cartográficos, cartas topográficas, zoneamento e mapeamento, a partir das imagens de satélite, auxiliam na caracterização, monitoramento e identificação de ameaças aos recursos naturais. Têm, portanto, papel fundamental e imprescindível nas geotecnologias e sensoriamento remoto na gestão ambiental e detecção de impactos (KUPLICH, 2003).

Portanto, gerir os elementos naturais do estado é indispensável para preservação da qualidade dos mesmos e de vida dos seres que deles dependem (SANTOS E LEAL, 2013).Para isso, dados georreferenciados, construções de mapas por integração de imagens de satélites, dentre outras técnicas de geoprocessamento, têm auxiliado na avaliação do potencial dos ecossistemas maranhenses, do seu monitoramento, caracterização e identificação da capacidade de suporte dos ambientes. A exemplo, imagens LandSat TM 5, cooperam para avaliação de potencialidade e fragilidade dos manguezais no município de Cururupu (COSTA, 2006).

Em Timom, para mapeamento dos conflitos de uso nas áreas de proteção permanente na microbacia do Riacho do Roncandor, foram utilizadas cartas topográficas e imagens de alta resolução espacial do Satélite GeoEye (CARDOSO E SABÓIA, 2013)

\section{MATERIAIS E MÉTODOS}

Localizado no extremo noroeste da Região Nordeste e com área de 331937,450 km², e 217 municípios, o estado do Maranhão é o segundo maior do nordeste e possui segunda maior costa litorânea brasileira, tendo parte dos biomas amazônico, cerrado, manguezais e matas dos cocais em seu território. É rico em biodiversidade e dispõe de grande variedade de recursos naturais, bem como várias bacias hidrográficas, diversidade de solo, fauna e flora. Para o estudo, trata-se da utilização de dados referentes a geotecnologias com o intuito de reconhecer as áreas prioritárias para conservação e a disposição hidrográfica, bem como analisar o potencial natural do estado a partir da construção de mapas resultantes do Macrozoneamento Econômico Ecológico do Maranhão (MacroZEE-MA).

O MacroZEE-MA, coordenado pela Embrapa Monitoramento por Statélite e solicitado pelo Governo do Estado do Maranhão, é uma ferramenta que objetiva planejar e ordenar o território maranhense associando as relações ambientais, econômicas e sociais de maneira harmoniosa, integrando as ações e políticas púbicas territoriais e articulando os interesses da 
sociedade civil à gestão do território. Possui uma série de dados cartográficos e numéricos que constituem sua base de dados e diretrizes metodológicas dividas em quatro fases que abrangem planejamento, diagnóstico, prognósticos e subsídios à implementação.

Constituído de dados inseridos a partir de um mesmo sistema de projeção (UTM, zona 23S, SIRGAS 2000), o banco de dados do MacroZEE-MA é subdividido em meio físicobiótico, socioeconômico e jurídico institucional. Através do conjunto de informações do meio físico-biótico obtém-se a caracterização do Maranhão e detecção dos seus sistemas naturais com suas limitações e potencialidades, sabendo que estão associadas a indicadores de perda de biodiversidade, vulnerabilidade à perda de solo, qualidade e quantidade dos recursos hídricos e recursos naturais e serviços ecossistêmicos disponíveis, respectivamente.

Para assegurar a atualização do plano de dados sobre utilização e cobertura da terra, vínculos às imagens de sensoriamento remoto foram indispensáveis. Obtidas e processadas do sensor remoto TM (Thematic Mapper - resolução temporal de 16 dias) a bordo do satélite Landsat 5, as imagens das 22 cenas entre 2006 e 2011 incluídas no banco de dados junto à geração do mosaico do Estado do Maranhão, deram suporte para elaboração do MacroZEEMA.

Figura 1 - Modelo conceitual do banco de dados e procedimentos para a realização do MacroZEE-MA.

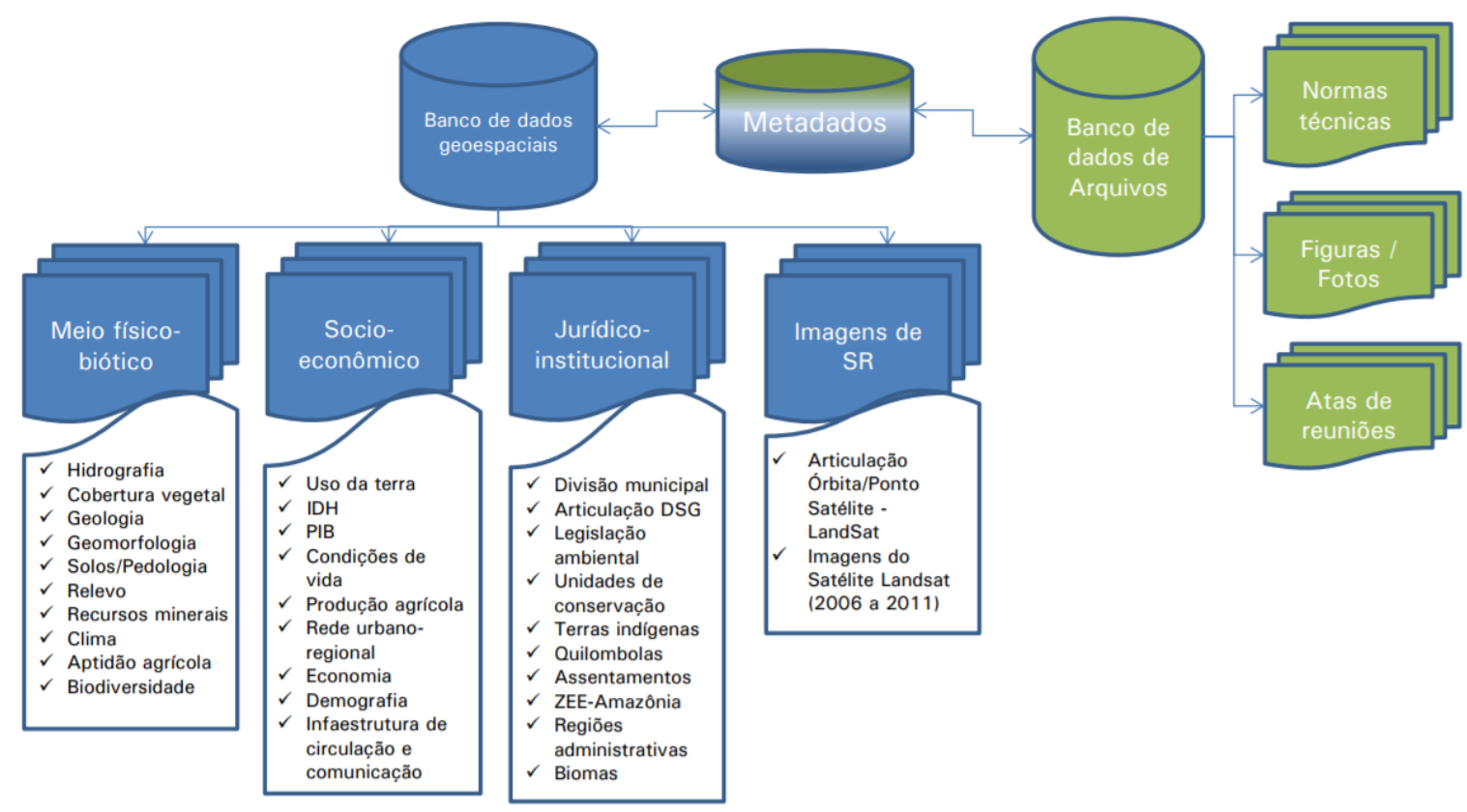

Fonte: Base de dados do MacroZee-MA 


\section{RESULTADOS E DISCUSSÕES}

Foram obtidos dados vetores e imagens. Os dados vetores compreendem áreas prioritárias de conservação, bacias hidrográficas, biomas, espécies ameaçadas, geologia, geomorfologia, hidrogeologia, solos e tipos de vegetação. Enquanto as imagens compreendem a elaboração do modelo digital do relevo. Verificou-se, conforme o grau de prioridade, as áreas com maior importância para gestão ambiental e as principais ações implementadas.

Figura 2 - Plano de Informação do MacroZEE-MA - Imagens de sensoriamento remoto
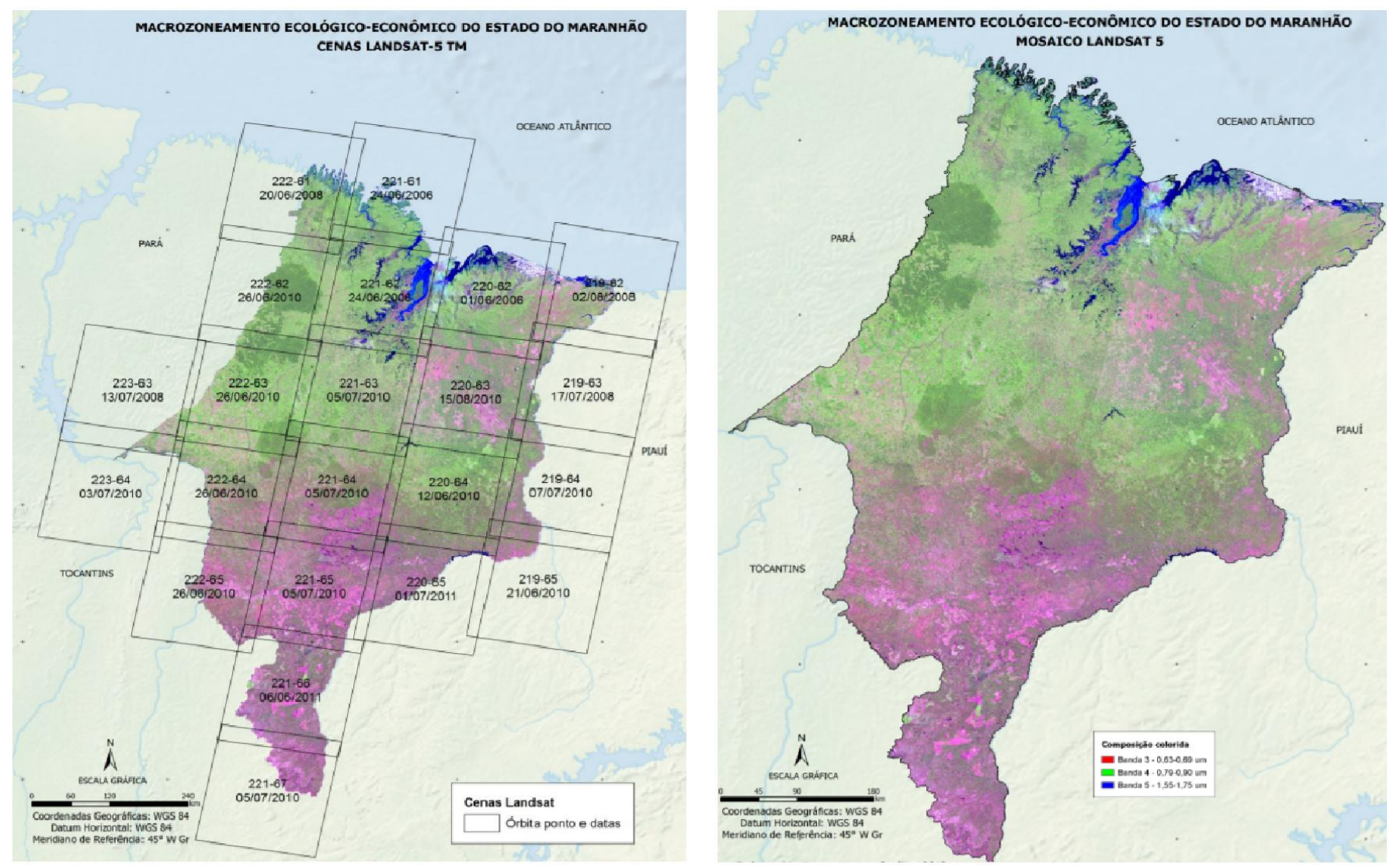

Fonte: Embrapa Monitoramento por Satélite, 2016.

Os dados resultantes do MacroZEE- MA destinam-se a avaliação das potencialidades e fragilidades dos recursos naturais, considerando suas limitações e disponibilidade frente ao uso dos serviços ecossistêmicos e apropriação de produtos da natureza. Dessa forma, torna-se possível a elaboração de alternativas possíveis para melhor gestão territorial em condições viáveis a um 
desenvolvimento sustentável articulando harmoniosamente sociedade, ambiente e economia.

Com a caracterização e diagnóstico da região, desenvolvimento de mapas e disposição de um banco de dados georreferenciados foi possível realizar a construção de mapas, provenientes do cruzamento dos dados resultantes do Macrozoneamento Econômico Ecológico do Maranhão.

Figura 3 - Uso dos dados do MacroZEE em um SIG

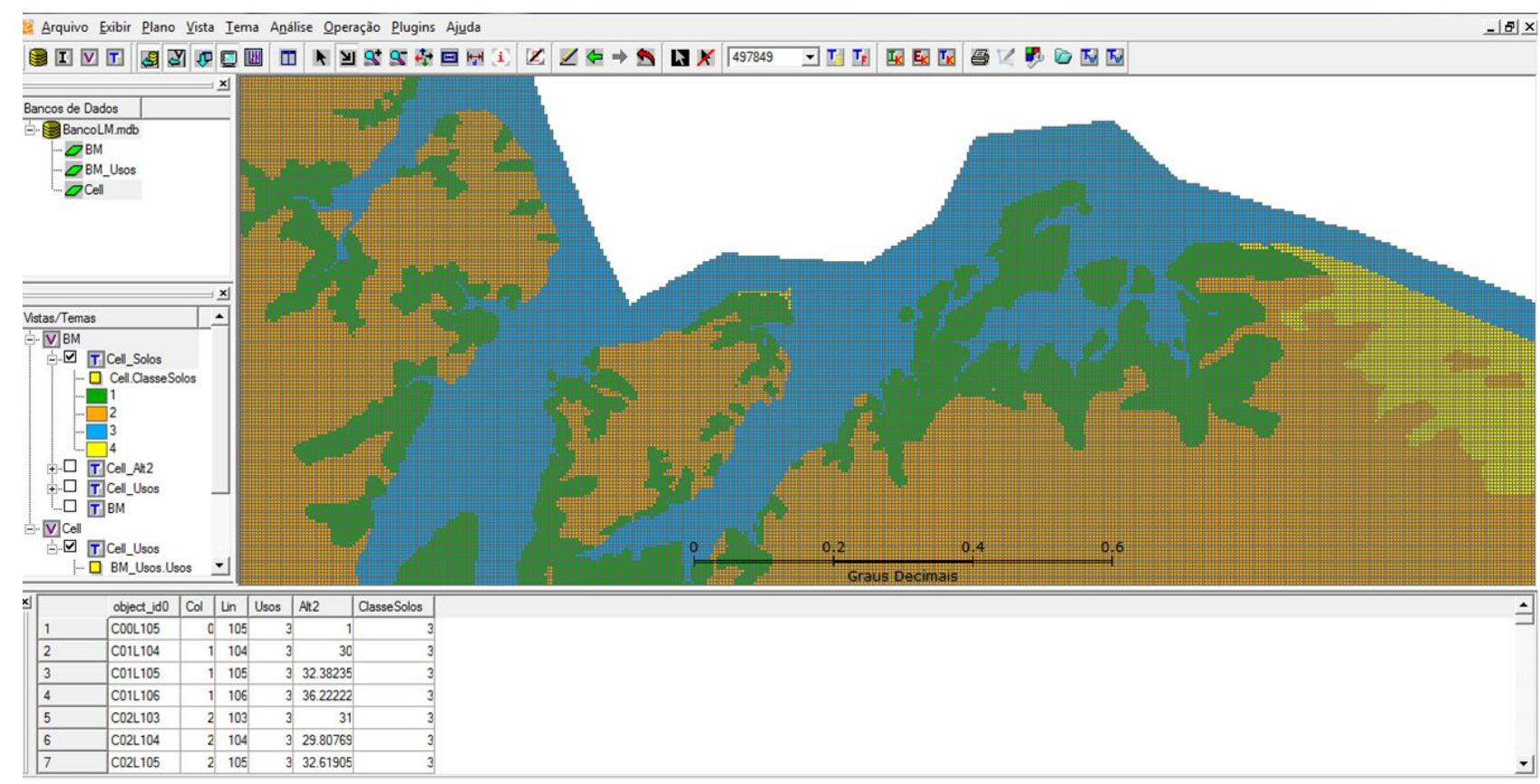

Fonte: Elaborada pelos autores.

A caracterização de solos (contida na imagem acima) é um fator extremamente relevante, portanto, indispensável para definição de áreas prioritárias para conservação, por exemplo. Sendo assim, os resultados facilitam a gestão e monitoramento do território maranhense, bem como dos recursos naturais que lhe garantem grande potencial.

\section{CONCLUSÃO}

Como ressalva Garcia e Leal 2012, o uso e manejo adequados dos recursos naturais devem ser práticas existentes para minimizar possíveis impactos que afetam o homem e sua qualidade de vida, bem como a dos outros seres. A partir de estudo aplicado baseado em sensoriamento remoto e técnicas de geoprocessamento, é possível propor ações mitigadoras para reprimir a degradação ambiental, recuperando ambientes degradados e promovendo o desenvolvimento sustentável regional, nacional e mundial.

Apesar da grande diversidade ecossistêmica do estado, é claramente possível realizar uma 
gestão ambiental para planejar, ordenar e assegurar o seu potencial junto à qualidade do meio harmonizando as relações e interesses sociais, econômicos e ambientais em torno dessa gestão. Temos bases de dados já disponibilizadas pela Empresa Brasileira de pecuária e agricultura (EMBRAPA) para isso. Logo, o manejo dos recursos deve ser dotado de gerenciamento visando o desenvolvimento sustentável e garantindo quantidade e qualidade dos recursos naturais, bem como maior aproveitamento do potencial nas possíveis destinações de uso.

\section{REFERÊNCIAS}

BOLFE, E. L. et al. Avaliação da classificação digital de povoamentos florestais em imagens de satélite através de índices de acurácia. Revista Árvore, Minas Gerais, v. 28, n.1, p. 85-90, 2004.

CAPOANI, V; SANTOS, D. R. Análise qualitativa do uso e ocupação da terra no assentamento alvorada, Júlio de Castilhos - Rio Grande Do Sul. Revista Nera, São Paulo, v. 15, n. 20, jan./jun. 2012.

CARDOSO, Josenete Assunção; SABÓIA, Cláudia Maria de Aquino. Mapeamento dos conflitos de uso nas áreas de preservação permanente (apps) da microbacia do Riacho do Roncador, Timon (MA). Boletim Goiano de Geografia, Goiânia, v. 33, n. 3, p. 477-492, set./dez. 2013.

COSTA, M. R. P. et al. Avaliação das potencialidades e fragilidades das áreas de manguezal para a implementação do ecoturismo usando ferramentas de sensoriamento remoto em Cururupu - MA, Brasil. Caminhos de Geografia, Universidade Federal de Uberlândia, p. 237-243, fev. 2006.

DULLEY, R. D. Noção de natureza, ambiente, meio ambiente, recursos ambientais e recursos naturais. Agrícola, São Paulo, v. 51, n. 2, p. 15-26, jul./dez. 2004

EMBRAPA MONITORAMENTO POR SATÉLITE. Zoneamento ecológico-econômico do

Estado do Maranhão: uso e ocupação do solo . Campinas: Embrapa Monitoramento por Satélite, 2013.

FILHO, P. W. M. S. F et al. Sensoriamento remoto e recursos naturais da Amazônia. Ciência e Cultura, v. 58, n. 3, São Paulo, jul./set. 2006. 
FUKS, Maurício. Indeterminação entrópica na economia: a exaustão de recursos naturais. Revista Brasileira de Economia, Rio de Janeiro, v.48, n.2, p.223-9, abr./jun. 1994.

GARCIA, Renata Marchi; LEAL, Antônio Cezar. Planejamento ambiental e gestão das águas: estudo aplicado à bacia hidrográfica do Ribeirão Taquaruçu, Sandovalina, São Paulo. Fórum Ambiental da Alta Paulista, v. 8, n.2, p. 86-102, 2012.

KUPLICH, T. M. Estudos florestais com imagens de radar. Espaço \& Geografia, v. 6, n. 1, 2003.

LEMOS, André Luiz Ferreira; SILVA, José de Arimatea. Desmatamento na Amazônia Legal: Evolução, Causas, Monitoramento e Possibilidades de Mitigação Através do Fundo Amazônia. Floresta e Ambiente, Universidade Federal Rural do Rio de Janeiro (UFRRJ), jan./mar. 2011.

MALTHUS, T. R. Um ensaio sobre o princípio da população. J. M. Dent, London, v. 2, p. 150, 1960.

MEDEIROS, Leonilde Servolo; LEITE, Sérgio. Formação dos assentados rurais no Brasil processos sociais e políticas públicas. Estudos Sociedade e Agricultura, Rio Grande do Sul, out. 1999.

NASCIMENTO, M.C. et al. Uso do geoprocessamento na identificação de conflito de uso da terra em áreas de preservação permanente na bacia hidrográfica do rio Alegre, Espírito Santo. Ciência Florestal, Santa Maria, v. 15, n. 2, p. 207-220, 2005.

SANTOS, Luiz Carlos Araujo; LEAL, Antonio Cezar. Gerenciamento de recursos hídricos no estado do Maranhão - Brasil. Observatorium: Revista Eletrônica de Geografia, Universidade Federal de Uberlândia, v.5, n.13, p. 39-65, jun. 2013.

SILVA, A. P. N. et al. Dinâmica espaço-temporal da vegetação no semi-árido de Pernambuco. Revista Caatinga, Mossoró, v.22, n.4, p.195-205, out./dez. 2009. 